\title{
EXISTENCE OF SOLUTIONS TO A ONE-DIMENSIONAL HAMILTON-JACOBI EQUATION WITH A DEGENERATE HAMILTONIAN
}

Abstract. We study a one-dimensional Hamilton-Jacobi initial value problem for a degenerate Hamiltonian occurring in multipeakon dynamics. Such a degenerate problem does not obey the usual viscosity solutions theory; viability type extensions do not seem to cover it either. Thanks to a particular change of variables, we reduce the problem to the case where Hopf theory is sufficient. Moreover, we show that our solutions are solutions in the viscosity sense.

1. Introduction. We consider the following Hamilton-Jacobi equation related to the multipeakon problem for the Camassa-Holm equation (see 2, 1, 9, or 8]):

$$
\begin{cases}u_{t}+\frac{1}{2}\left\langle\nabla u, E_{n} \nabla u\right\rangle=0, & t>0, x \in \mathbb{R}^{n}, \\ u(0, x)=u_{0}(x), & x \in \mathbb{R}^{n},\end{cases}
$$

where the metric $E_{n}=E_{n}(x)\left(x \in \mathbb{R}^{n}\right)$ is given by

$$
\begin{aligned}
E_{n}(x) & =\left(e^{-\left|x_{j}-x_{k}\right|}\right)_{j, k=1}^{n} \\
& =\left(\begin{array}{ccccc}
1 & e^{-\left|x_{1}-x_{2}\right|} & \ldots & \cdots & e^{-\left|x_{1}-x_{n}\right|} \\
e^{-\left|x_{2}-x_{1}\right|} & \cdots & & & \vdots \\
\vdots & & & \cdots & e^{-\left|x_{n-1}-x_{n}\right|} \\
e^{-\left|x_{n}-x_{1}\right|} & \cdots & \cdots & e^{-\left|x_{n}-x_{n-1}\right|} & 1
\end{array}\right) .
\end{aligned}
$$

2020 Mathematics Subject Classification: 35F21, 35C99, 35D40.

Key words and phrases: Hopf-Lax formula, Hamilton-Jacobi equations, viscosity solution. Received 24 February 2020; revised 30 May 2020.

Published online 19 October 2020. 
Notice that such a matrix is singular at $x_{i}=x_{j}$, while for $x_{i} \neq x_{j}$ it is invertible and the inverse can be explicitly computed [3. However, the singularity at $x_{i}=x_{j}$ leads to an interesting problem, which does not seem to be covered by the general theory. Indeed, the inverse of $E$ cannot be computed for $x_{i}=x_{j}$ and the Lagrangian is not computable at such points [3]. Though some extensions of the theory, utilizing differential inclusions and the viability method, to Lagrangians which may be infinite at some points are available (see [6] or [7] for instance), our case violates the assumptions imposed on the Hamiltonians in such cases. For example, one can check that already a two-dimensional Hamiltonian, corresponding to a two-peakon, violates assumption (A2) in [7], which is a special kind of sub-Lipschitz behavior of the epigraphical mapping of the Hamiltonian.

In the present paper we focus on the one-dimensional simplification of the system, still including the degeneracy. We shall introduce a change of variables leading to a concept of solution via an extension of the Hopf-Lax formula [5]. Next, we show that one cannot expect such a change of variables in higher dimensions, which will be studied in a forthcoming paper of the first author, where viscosity solutions will be applied. Actually, our solution of the one-dimensional simplification will also be shown to be a viscosity solution in the sense of [4. Some regularity results in the present $1 \mathrm{~d}$ case will give us hints concerning the regularity and uniqueness in higher dimensions.

Let us describe in detail the problem we are dealing with in the present note. The initial value problem of the following form is studied:

$$
\begin{cases}u_{t}+\frac{1}{2} H\left(x, u_{x}\right)=0, & t>0, x \in \mathbb{R}, \\ u(0, x)=u_{0}(x), & x \in \mathbb{R},\end{cases}
$$

where the Hamiltonian $H\left(x, u_{x}\right)$ is given by

$$
H\left(x, u_{x}\right)=\frac{1}{2}|x| u_{x}^{2}=\frac{1}{2}\left(\sqrt{|x|} u_{x}\right)^{2}
$$

or

$$
H\left(x, u_{x}\right)=\frac{1}{2}\left(1-e^{-|x|}\right) u_{x}^{2}=\frac{1}{2}\left(\sqrt{1-e^{-|x|}} u_{x}\right)^{2} .
$$

We shall construct solutions to 1.2 with these Hamiltonians.

2. Construction of a solution. Our solution is obtained via a change of variables which transforms 1.2 to a problem in which a solution is given via the Hopf-Lax formula. As we shall see, it works only in dimension 1.

TheOREm 2.1. Let $H=H\left(x, u_{x}\right)$ be a Hamiltonian given by 1.3 or 1.4. Assume that the initial datum $u_{0}(x)$ is a compactly supported $C^{1}$ function. Then there exists a solution $u=u(t, x)$ such that $u(\cdot, x)$ is continuous for all $x \in \mathbb{R}$ and for any $t>0, u(t, \cdot)$ is $\frac{1}{2}$-Hölder continuous and differentiable a.e. Moreover, the solution is locally Lipschitz continuous except 
at $x=0$ and the following representation formulas hold: If the Hamiltonian $H$ is given by (1.3), then

$$
u(t, x)=\inf _{y \in \mathbb{R}}\left\{\frac{|A(x)-y|^{2}}{2 t}+u_{0}\left(A^{-1}(y)\right)\right\},
$$

while for $H$ given by (1.4),

$$
u(t, x)=\inf _{y \in \mathbb{R}}\left\{\frac{|B(x)-y|^{2}}{2 t}+u_{0}\left(B^{-1}(y)\right)\right\},
$$

where the functions $A(x)$ and $B(x)$ are given below.

Proof. Let $A=A(x)$ and $B=B(x)$ be defined by

$$
\begin{aligned}
& A(x)= \begin{cases}2 \sqrt{x}, & x \geq 0, \\
-2 \sqrt{-x}, & x<0,\end{cases} \\
& B(x)= \begin{cases}\log \left(\frac{1+\sqrt{1-e^{-x}}}{1-\sqrt{1-e^{-x}}}\right), & x \geq 0, \\
-\log \left(\frac{1+\sqrt{1-e^{x}}}{1-\sqrt{1-e^{x}}}\right), & x<0 .\end{cases}
\end{aligned}
$$

These functions are bijective, and thus we can find a point $x=A^{-1}(z)$ or $x=B^{-1}(z)$ for any $z \in \mathbb{R}$. Namely,

$$
A^{-1}(z)=\left\{\begin{array}{ll}
\frac{1}{4} z^{2}, & z \geq 0, \\
-\frac{1}{4} z^{2}, & z<0,
\end{array} \quad B^{-1}(z)= \begin{cases}-\log \left[\frac{4 e^{z}}{\left(e^{z}+1\right)^{2}}\right], & z \geq 0, \\
\log \left[\frac{4 e^{z}}{\left(e^{z}+1\right)^{2}}\right], & z<0 .\end{cases}\right.
$$

We first consider the case (1.3). Then the function $v(t, A(x))=u(t, x)$ satisfies

$$
u_{x}(t, x)=\left.A_{x}(x) v_{z}(t, z)\right|_{(t, z)=(t, A(x))}=\left.\frac{1}{\sqrt{|x|}} \operatorname{sgn}(x) v_{z}(t, z)\right|_{(t, z)=(t, A(x))},
$$

and thus

$$
\left.v_{z}(t, z)\right|_{(t, z)=(t, A(x))} ^{2}=\left(\sqrt{|x|} u_{x}\right)^{2} .
$$

Therefore we may reduce our problem to the following well-known problem:

$$
\begin{cases}v_{t}+\frac{1}{2} v_{z}^{2}=0, & t>0, z \in \mathbb{R}, \\ v(0, z)=v_{0}(z), & x \in \mathbb{R} .\end{cases}
$$

When the Hamiltonian $H$ is given by (1.4), we observe $u_{x}(t, x)=\left.B^{\prime}(x) v_{z}(t, z)\right|_{(t, z)=(t, B(x))}=\left.\frac{1}{\sqrt{1-e^{-|x|}}} \operatorname{sgn}(x) v_{z}(t, z)\right|_{(t, z)=(t, B(x))}$.

Thus we have

$$
\left.v_{z}(t, z)\right|_{(t, z)=(t, B(x))} ^{2}=\left(\sqrt{1-e^{-|x|}} u_{x}(t, x)\right)^{2} .
$$


The above observation implies that equation 1.2 with the Hamiltonian $H\left(x, u_{x}\right)=\left(1-e^{-|x|}\right) u_{x}^{2}$ also reduces to 2.5$)$. It is well-known (see for instance [5]) that the Hopf-Lax formula gives us a unique viscosity solution to (2.5) as follows if $v_{0}=v_{0}(x)$ is a given Lipschitz function:

$$
v(t, z)=\min _{y \in \mathbb{R}}\left\{\frac{(z-y)^{2}}{2 t}+v_{0}(y)\right\} .
$$

And our $v_{0}$ is global Lipschitz continuous since $u_{0} \in C_{0}^{1}(\mathbb{R})$ and $A^{-1}, B^{-1}$ are local Lipschitz continuous.

Since the function $v=v(t, z)$ given by (2.6) is Lipschitz continuous on $[0, \infty) \times \mathbb{R}$ and $\lim _{t \rightarrow 0} v(t, z)=v_{0}(z)$, we observe that $v$ solves the equation $v_{t}+\frac{1}{2}\left|v_{x}\right|^{2}=0$ almost everywhere in $(0, \infty) \times \mathbb{R}$. The changes of variable $x \mapsto A(x)$ and $x \mapsto B(x)$ are bijective, so the problem $(1.2)$ is reduced to (2.5). Finally, $u=u(t, \cdot)$ is $\frac{1}{2}$-Hölder continuous for all $t>0$.

We notice that we had some freedom in our choice of bijections $A$ and $B$. Namely, we could choose both $A$ or $-A$ as a change of variables, and in the same way we could choose $B$ or $-B$. Both choices lead to the same solution, as stated in the remark below.

REMARK 2.2. Let us consider the transformations

$$
\widetilde{A}(x)=-A(x), \quad \widetilde{B}(x)=-B(x) .
$$

Next, define $u_{1}(t, x)=v(t, \widetilde{A}(x)), z_{1}(t, x)=v(t, \widetilde{B}(x))$. The functions $u_{1}(t, x)$ and $z_{1}$ solve (1.2) with Hamiltonians (1.3) and 1.4 respectively, with initial data $u_{0,1}(x)=v_{0}(\widetilde{A}(x))$ and $z_{0,1}(x)=v_{0}(\widetilde{B}(x))$. We claim that the solutions $u(t, x)=v(t, A(x))$ and $u_{1}(t, x)$ coincide. The same holds for $z(t, x)=v(t, B(x))$ and $z_{1}$.

Proof. Indeed, $u_{0}(z)=v_{0}(A(x))$ and $u_{0,1}(z)=v_{0}(-A(x))$, and the Hopf-Lax formula gives

$$
\begin{aligned}
u(t, x)=v(t, A(x)) & =\min _{y \in \mathbb{R}}\left\{\frac{|A(x)-y|^{2}}{2 t}+u_{0}\left(A^{-1}(y)\right)\right\} \\
& =\min _{y \in \mathbb{R}}\left\{\frac{|-A(x)-(-y)|^{2}}{2 t}+u_{0}\left(A^{-1}(-(-y))\right)\right\} \\
& =\min _{\bar{y} \in \mathbb{R}}\left\{\frac{|-A(x)-\bar{y}|^{2}}{2 t}+u_{0}\left(-A^{-1}(\bar{y})\right)\right\} \\
& =\min _{\bar{y} \in \mathbb{R}}\left\{\frac{|-A(x)-\bar{y}|^{2}}{2 t}+u_{0}\left((-A)^{-1}(\bar{y})\right)\right\} \\
& =v(t,-A(x))=u_{1}(t, x) .
\end{aligned}
$$

The same argument holds for $B(x)$ and $\widetilde{B}(x)$. 
The remaining question is uniqueness of our solution. On the one hand, in the next section, we prove that it is a viscosity solution of (1.2). Moreover, as we already know, our solution $u$ is in the regularity class

$$
\begin{aligned}
\mathcal{A}:=\{f:[0, T] \times \mathbb{R} \rightarrow & \mathbb{R} \mid \text { for every } x \in \mathbb{R}, f(\cdot, x) \in \operatorname{Lip}([0, T]), \\
& \text { for every } \left.t>0, f(t, \cdot) \in \operatorname{Lip}_{\text {loc }}(\mathbb{R} \backslash\{0\}) \cap C^{1 / 2}(\mathbb{R})\right\} .
\end{aligned}
$$

Hence $u$ is differentiable almost everywhere in $[0, T] \times \mathbb{R}$.

The uniqueness of a viscosity solution in such a regularity class is a question we have to leave open. Let us mention, however, that defining $v(t, z)=u\left(t, A^{-1}(z)\right)$ in the case of 1.3$)$, or $v(t, z)=u\left(t, B^{-1}(z)\right)$ when $H$ is given by (1.4) (in both cases $v_{0}$ is a Lipschitz continuous function), we see that, in both cases, $v$ is a solution to [2.5). By [5, Lemma 3.3.4], we know that $v$, given by the Hopf-Lax formula, is a semiconcave function for any $t>0$. Then, due to [5, Theorem 3.3.7], $v=v(t, z)$ is a unique weak solution of (2.5) in the class of semiconcave functions. So our solution $u$ corresponds to the unique solution $v$ (given by the Hopf-Lax formula) of 2.5. This correspondence allows us to hope for the uniqueness of solution in the class $\mathcal{A}$.

2.1. Invalidity of a construction in higher dimensional cases. Let us explain briefly why the above idea does not work in higher dimensions and other techniques are necessary. To follow a similar approach in higher dimensions we would need a function $\varphi=\varphi(x) \in \mathbb{R}^{n}$ which would allow us to define

$$
u(t, x)=v(t, \varphi(x)),
$$

where $v$ would be a solution of the following simplified problem:

$$
\begin{cases}v_{t}+\frac{1}{2}\left|\nabla_{z} v\right|^{2}=0, & t>0, z \in \mathbb{R}^{n}, \\ v(0, z)=z_{0}(z), & z \in \mathbb{R}^{n} .\end{cases}
$$

Then, in particular, we would have

$$
\frac{\partial u}{\partial x_{j}}=\frac{\partial \varphi_{1}}{\partial x_{j}} \frac{\partial v}{\partial z_{1}}+\frac{\partial \varphi_{2}}{\partial x_{j}} \frac{\partial v}{\partial z_{2}}+\cdots+\frac{\partial \varphi_{n}}{\partial x_{j}} \frac{\partial v}{\partial z_{n}},
$$

and consequently

$$
\left(\begin{array}{c}
\frac{\partial u}{\partial x_{1}}(t, x) \\
\frac{\partial u}{\partial x_{2}}(t, x) \\
\vdots \\
\frac{\partial u}{\partial x_{n}}(t, x)
\end{array}\right)=\left.\left(\begin{array}{cccc}
\frac{\partial \varphi_{1}}{\partial x_{1}}(x) & \frac{\partial \varphi_{2}}{\partial x_{1}}(x) & \cdots & \frac{\partial \varphi_{n}}{\partial x_{1}}(x) \\
\frac{\partial \varphi_{1}}{\partial x_{2}}(x) & \frac{\partial \varphi_{2}}{\partial x_{2}}(x) & \cdots & \frac{\partial \varphi_{n}}{\partial x_{2}}(x) \\
\vdots & \vdots & \ddots & \vdots \\
\frac{\partial \varphi_{1}}{\partial x_{n}}(x) & \frac{\partial \varphi_{2}}{\partial x_{n}}(x) & \cdots & \frac{\partial \varphi_{n}}{\partial x_{n}}(x)
\end{array}\right)\left(\begin{array}{c}
\frac{\partial v}{\partial z_{1}}(t, z) \\
\frac{\partial v}{\partial z_{2}}(t, z) \\
\vdots \\
\frac{\partial v}{\partial z_{n}}(t, z)
\end{array}\right)\right|_{(t, z)=(t, \varphi(x))}
$$


So the Jacobi matrix

$$
\frac{\partial\left(\varphi_{1}, \ldots, \varphi_{n}\right)}{\partial\left(x_{1}, \ldots, x_{n}\right)}=\left(\frac{\partial \varphi_{j}}{\partial x_{k}}(x)\right)_{j, k=1}^{n}=\left(\sqrt{E_{n}}\right)^{-1}
$$

would have to exist. In order to show that this is impossible, let us analyze the case $n=2$. Then

$$
E_{2}(x)=\left(\begin{array}{cc}
1 & e^{-\left|x_{1}-x_{2}\right|} \\
e^{-\left|x_{1}-x_{2}\right|} & 1
\end{array}\right)
$$

and we observe

$$
\begin{aligned}
& \left(\sqrt{E_{2}(x)}\right)^{-1} \\
& =\frac{1}{2}\left(\begin{array}{ll}
\frac{1}{\sqrt{1-e^{-\left|x_{1}-x_{2}\right|}}}+\frac{1}{\sqrt{1+e^{-\left|x_{1}-x_{2}\right|}}} & \frac{1}{\sqrt{1+e^{-\left|x_{1}-x_{2}\right|}}}-\frac{1}{\sqrt{1-e^{-\left|x_{1}-x_{2}\right|}}} \\
\frac{1}{\sqrt{1+e^{-\left|x_{1}-x_{2}\right|}}}-\frac{1}{\sqrt{1-e^{-\left|x_{1}-x_{2}\right|}}} & \frac{1}{\sqrt{1-e^{-\left|x_{1}-x_{2}\right|}}}+\frac{1}{\sqrt{1+e^{-\left|x_{1}-x_{2}\right|}}}
\end{array}\right) .
\end{aligned}
$$

Now we notice that the rows of the above matrix are not gradients of potentials, so $\frac{\partial\left(\varphi_{1}, \varphi_{2}\right)}{\partial\left(x_{1}, x_{2}\right)} \neq\left(\sqrt{E_{2}(x)}\right)^{-1}$. Hence the reduction of our original problem to 2.8 is impossible already for $n=2$.

3. Viscosity solutions. In this section, we show that the solutions defined via (2.1) and $\left(2.2\right.$ are actually viscosity solutions. Since $A^{-1}$ as well as $B^{-1}$ are not $C^{\infty}$ functions, we need some additional work to justify this fact. Indeed, both $A^{-1}$ and $B^{-1}$ are $C^{1}$ functions, with derivatives $\left(A^{-1}\right)^{\prime}(z)=2|z|$ and $\left(B^{-1}\right)^{\prime}(z)=\operatorname{sgn}(z) \frac{e^{z}-1}{e^{z}+1}$. But, as one sees, the second derivatives are both discontinuous at 0 . In the classical definition of viscosity solutions test functions are taken $C^{\infty}$ regular [4. The lack of such regularity of $A^{-1}$ and $B^{-1}$ requires some preparatory steps. First, notice that in 5 , Theorem 10.3.3] it is proven that the Hopf-Lax formula defines a viscosity solution of a Hamilton-Jacobi equation. We notice that the proof of the claim in 5] works even if test functions are taken only $C^{1}$ regular. Indeed, that proof yields the following more general lemma.

\section{Lemma 3.1. Consider a Hamilton-Jacobi initial value problem}

$$
\begin{cases}u_{t}+\frac{1}{2} H(x, \nabla u)=0, & t>0, x \in \mathbb{R}^{n}, \\ u(0, x)=u_{0}(x), & x \in \mathbb{R}^{n},\end{cases}
$$

where $H$ is a $C^{2}$ function, convex in the second variable. Moreover, suppose that for each $x \in \mathbb{R}$,

$$
\lim _{p \rightarrow \infty} \frac{H(x, p)}{|p|}=\infty
$$


Then $u$, given by the Hopf-Lax formula, is a viscosity solution with a $C^{1}$ test function $\phi$. More precisely, for any $\phi \in C^{1}\left((0, \infty) \times \mathbb{R}^{n}\right)$ and any point $\left(t_{0}, x_{0}\right)$ at which $u-\phi$ admits a local minimum we have

$$
\phi_{t}\left(t_{0}, x_{0}\right)-H\left(x_{0}, \nabla_{x} \phi\left(t_{0}, x_{0}\right)\right) \geq 0,
$$

so $u$ is a viscosity supersolution to (3.1). Moreover, for any $\phi \in$ $C^{1}\left((0, \infty) \times \mathbb{R}^{n}\right)$ and any point $\left(t_{0}, x_{0}\right)$ at which $u-\phi$ admits a local maximum we have

$$
\phi_{t}\left(t_{0}, x_{0}\right)-H\left(x_{0}, \nabla_{x} \phi\left(t_{0}, x_{0}\right)\right) \leq 0,
$$

so $u$ is a viscosity subsolution. Altogether, $u$ is a viscosity solution of (3.1) with test functions $\phi$ only $C^{1}$ regular.

By decreasing the regularity of test functions in the previous lemma, we can avoid technical difficulties stemming from the fact that $A^{-1}$ and $B^{-1}$ are not $C^{\infty}$ and prove the main result of this section.

Proposition 3.2. The function u given by 2.1) defines a viscosity solution to 1.2 with Hamiltonian $H$ given by 1.3 . Similarly, formula 2.2 defines a viscosity solution to (1.2), 1.4.

Proof. First consider $u=u(t, x)$ given by (2.1). We start by showing that $u$ is a viscosity subsolution. To this end we have to check that for arbitrary $C^{\infty}$ function $\phi=\phi(t, x)$ for which $u-\phi$ achieves a local maximum at a point $\left(t_{0}, x_{0}\right) \in(0, \infty) \times \mathbb{R}$, we have

$$
\phi_{t}\left(t_{0}, x_{0}\right)+\frac{1}{2}\left|x_{0}\right|\left|\phi_{x}\left(t_{0}, x_{0}\right)\right|^{2} \leq 0 .
$$

Due to the low regularity of $A^{-1}$, in the first step we show (3.4) for a wider class of $C^{1}$ functions. Define $\tilde{\phi}(t, z):=\phi\left(t, A^{-1}(z)\right)$. As previously, $v(t, z)=$ $u\left(t, A^{-1}(z)\right)$. We observe that $v-\tilde{\phi}$ has a local maximum at $\left(t_{0}, z_{0}\right)=$ $\left(t_{0}, A\left(x_{0}\right)\right)$. Moreover, $v$ given by the Hopf-Lax formula solves 2.5), so in view of Lemma 3.1, $v(t, z)$ is a viscosity solution to (2.5). Hence, by (3.3), at each $\left(t_{0}, z_{0}\right)$ at which $v-\tilde{\phi}$ has a maximum we have

$$
\tilde{\phi}_{t}\left(t_{0}, x_{0}\right)-\frac{1}{2}\left|\tilde{\phi}_{z}\left(t_{0}, z_{0}\right)\right|^{2} \leq 0 .
$$

Consequently,

$$
\phi_{t}\left(t_{0}, x_{0}\right)+\frac{1}{2}\left|x_{0}\right|\left|\phi_{x}\left(t_{0}, x_{0}\right)\right|^{2} \leq 0 .
$$

Now, take $\phi \in C^{\infty}$ such that $u-\phi$ achieves a local maximum at $\left(t_{0}, x_{0}\right)$. The function $\phi$ is in particular $C^{1}$ and by the previous step (3.4) holds, so $u$ is a viscosity subsolution. In order to show that $u$ is also a viscosity supersolution, again we start with $\phi \in C^{1}$ such that $u-\phi$ admits a local minimum at $\left(t_{0}, x_{0}\right)$. Once more we define $v(t, z)=u\left(t, A^{-1}(z)\right)$ and $\tilde{\phi}(t, z):=\phi\left(t, A^{-1}(z)\right)$. Due 
to Lemma 3.1, the function $v$ given by the Hopf-Lax formula solves 2.5 in the viscosity sense. In particular, it is a supersolution to 2.5. Next, (3.2) allows us to write

$$
\tilde{\phi}_{t}\left(t_{0}, z_{0}\right)-\frac{1}{2}\left|\tilde{\phi}_{z}\left(t_{0}, z_{0}\right)\right|^{2} \geq 0
$$

for $\left(t_{0}, z_{0}\right)$ being a minimum point of $v-\tilde{\phi}$. Again, this gives us

$$
\phi_{t}\left(t_{0}, x_{0}\right)+\frac{1}{2}\left|x_{0}\right|\left|\phi_{x}\left(t_{0}, x_{0}\right)\right|^{2} \leq 0 .
$$

The latter formula holds in particular for $\phi \in C^{\infty}$, so $u$ is also a viscosity supersolution. We conclude that $u$ given by (2.1) is a viscosity solution of $(1.2),(1.3)$.

To complete the proof of Proposition 3.2 we still need to show that $u$ given by (2.2) is a viscosity solution to (1.2), (1.4).

The proof is exactly the same. We define $v(t, z)=u\left(t, B^{-1}(z)\right)$ and $\tilde{\phi}(t, z)=\phi\left(t, B^{-1}(z)\right)$ for any $z \in \mathbb{R}$. The function $\tilde{\phi}$ is a $C^{1}$ function (as a composition of a smooth function $\phi$ and a $C^{1}$ bijection $B^{-1}$ ), but it may not be $C^{\infty}$. As previously, we use Lemma 3.1 for $C^{1}$ test functions and show that $v$ is both a viscosity sub- and supersolution. In particular, it is a viscosity solution in the usual sense [4, with $C^{\infty}$ test functions.

Acknowledgements. The first author is grateful to Andrzej Święch and Sławomir Plaskacz for helpful discussions.

T.C. was supported by the National Science Center of Poland grant SONATA BIS 7, no. UMO-2017/26/E/ST1/00989. During the preparation of this paper the second author was supported by the National Science Center of Poland grant no. 2016/23/B/ ST1/00434 at the University of Wrocław. This work was partially supported by the grant 346300 for IMPAN from the Simons Foundation and the matching 2015-2019 Polish MNiSW fund.

\section{References}

[1] R. Beals, D. Sattinger and J. Szmigielski, Multipeakons and the classical moment problem, Adv. Math. 154 (2000), 229-257.

[2] R. Camassa and D. Holm, An integrable shallow water equation with peaked solitons, Phys. Rev. Lett. 71 (1993), 1661-1664.

[3] T. Cieślak, M. Gaczkowski, M. Kubkowski and M. Małogrosz, Multipeakons viewed as geodesics, Bull. Polish Acad. Sci. 65 (2017), 153-164.

[4] M. G. Crandall, L. C. Evans and P.-L. Lions, Some properties of viscosity solutions of Hamilton-Jacobi equations, Trans. Amer. Math. Soc. 282 (1984), 487-502.

[5] L. C. Evans, Partial Differential Equations, 2nd ed., Amer. Math. Soc., 2010.

[6] H. Frankowska, S. Plaskacz and T. Rzeżuchowski, Measure viability theorems and the Hamilton-Jacobi-Bellman equation, J. Differential Equations 116 (1995), 265-305.

[7] G. N. Galbraith, Extended Hamilton-Jacobi characterization of value functions in optimal control, SIAM J. Control Optim. 39 (2000), 281-0305. 
[8] K. Grunert and H. Holden, The general peakon-antipeakon solution for the CamassaHolm equation, J. Hyperbolic Differential Equations 13 (2016), 353-380.

[9] W. Kryński, Dissipative prolongations of the multipeakon solutions to the CamassaHolm equation, J. Differential Equations 266 (2019), 1832-1850.

Tomasz Cieślak

Institute of Mathematics

Polish Academy of Sciences

00-656 Warszawa, Poland

ORCID: 0000-0003-4191-4231

E-mail: cieslak@impan.pl
Hiroshi Wakui

Mathematical Institute

University of Wrocław pl. Grunwaldzki 2/4 50-384 Wrocław, Poland and Faculty of Science Division I Tokyo University of Science Tokyo 162-8601, Japan E-mail: hiroshi.wakui@rs.tus.ac.jp 
